\title{
Properties of Highly Transparent Conductive Ga-Doped ZnO Films prepared on Polymer Substrates by Reactive Plasma Deposition with DC Arc Discharge
}

\author{
Aki Miyake, Takahiro Yamada, Hisao Makino, Naoki Yamamoto and Tetsuya Yamamoto \\ Materials Design Center, Research Institute, Kochi Univ. Tech., \\ 185 Miyanokuchi, Tosayamada-cho, Kami-shi, Kochi 782-8502, Japan
}

\begin{abstract}
Highly transparent conductive polycrystalline Ga-doped $\mathrm{ZnO}$ (GZO) films with a thickness of about $100 \mathrm{~nm}$ prepared on cyclo-olefin polymer (COP) or glass substrates at various temperatures below $90^{\circ} \mathrm{C}$ by ion plating with DC-arc discharge were investigated. A systematic study has been made of the influence of substrate temperature $T_{\mathrm{s}}$ on the structural, electrical, optical and residual stress properties of GZO films. An increase in $\mathrm{T}_{\mathrm{s}}$ resulted in a beneficial effect on the crystallinity, electrical conductivity and transmission in the visible (VIS) range regardless of the type of substrate. The minimum resisitivity for GZO on glass and COP substrates was as low as $5.0 \mu \Omega \mathrm{m}$ and $5.8 \mu \Omega \mathrm{m}$, respectively. For all the GZO films with average optical transmission above $90 \%$ in the VIS region, with increasing $\mathrm{T}_{\mathrm{s}}$, the optical absorption loss in the visible range decreased according in order of increasing Hall mobility. All the GZO films were found to be compressively stressed. The residual stresses, evaluated from the XRD spectrum, in GZO films on glass substrates are a decreasing function of $T_{s}$, whereas they are an increasing function of $T_{s}$ for GZO films on COP substrates. This finding indicates that for the GZO films on COP substrates deposited at higher temperature, thermal stress is dominant.
\end{abstract}

Keyword: Ga-doped zinc oxide, transparent conductive oxide, cyclo-olefin polymer

\section{Introduction}

Highly transparent conducting oxide (TCO) films on polymer substrates [1-4] have recently been attracting considerable attention for use in electrodes in mobile devices, such as mobile phones and the emerging flexible liquid crystal displays (LCDs) and organic light-emitting diodes (OLEDs) [5]. The advantages of polymer substrates over glass substrates include light weight, compactness, robustness, impact resistance and flexibility. However, polymer substrates also have disadvantages such as low heat resistance and a large thermal expansion coefficient compared with glass substrates.

There are many methods of fabricating TCO films on polymer substrates. The most straightforward method is to fabricate TCO directly on polymer substrates. The advantages of this method are low cost and the ability to make large-area flexible structures. Nevertheless, because of the temperature limit imposed by the polymer substrates, many high-temperature processes are ruled out and the material properties cannot be optimized.

When the substrate is heated, the TCO films on it are subjected to the stress. This may cause both desirable and undesirable consequences:1) the film quality is improved by heating the substrate during the deposition of the film on the substrate; 2) the TCO may crack owing to the difference in the thermal expansion coefficient between the film 
and the substrate. Thus, it is very important not only to choose a material with acceptable crystallinity, even for growth at the low substrate temperature $T_{s}$, but also to develop a deposition process to obtain TCO films with good characteristics on polymer substrates with low $\mathrm{T}_{\mathrm{s}}$.

In our previous work [3], we reported the thickness dependence of the structural, electrical and optical properties of highly transparent conductive Ga-doped $\mathrm{ZnO}$ (GZO) films prepared directly on cyclo-olefin polymer (COP: ZEONOR by Nippon Zeon) substrates. Polycrystalline GZO films with different thicknesses in the range from 11 to $112 \mathrm{~nm}$ were prepared by ion-plating deposition with direct-current (DC) arc discharge. No intentional heating was applied to the substrate. The typical deposition rate was about $100 \mathrm{~nm} / \mathrm{min}$. $T_{s}$ was evaluated to be less than $100^{\circ} \mathrm{C}\left(<T_{g}\right.$ : glass transition temperature of COP, $163^{\circ} \mathrm{C}$ ). Under such deposition conditions, polycrystalline crack-free GZO films with good adherence to COP substrates and a (002) preferred orientation were obtained.

COPs have attractive properties, such as high transparency (total light transmittance, $T_{\text {total }}=92 \%$; refractive index at $589.3 \mathrm{~nm}, n_{d}=1.53$ ), high chemical and heat resistance $\left(T_{g}, 163^{\circ} \mathrm{C}\right.$ [6]) equivalent to that of engineering plastics, and water absorption of less than $0.01 \%$ (compared with polycarbonate (PC) $0.2 \%$ and polymethylmethacrylate (PMMA) 0.3\%).

Note that $T_{s}$, which strongly affects the TCO film properties, can be controlled by the exposure time to the arc plasma and must be optimized to realize crack-free highly transparent conductive GZO films.

Thus, in this study, we developed a multiple deposition method for intentionally achieving different values of $T_{s}$ lower than $100^{\circ} \mathrm{C}\left(<T_{g}\right)$. The aim of this study is to investigate the effects of $T_{s}$ on GZO film characteristics, such as structural, electrical, optical and residual stress properties, in order to clarify the issues to be resolved to enable a wide variety of applications.

\section{Experimental}

GZO films were deposited on COP substrates with a thickness of $2 \mathrm{~mm}$ or an alkali-free alumino silicate glass (HOYA NA-32) by ion-plating deposition with DC arc discharge [3,4,7-10].

Figure 1 shows a schematic diagram of the deposition system that enables the deposition of GZO films at a high rate (typically $100 \mathrm{~nm} / \mathrm{min}$ in this study, $170 \mathrm{~nm} / \mathrm{min}$ at $T_{s}=200^{\circ} \mathrm{C}$ ) $[3,4]$.

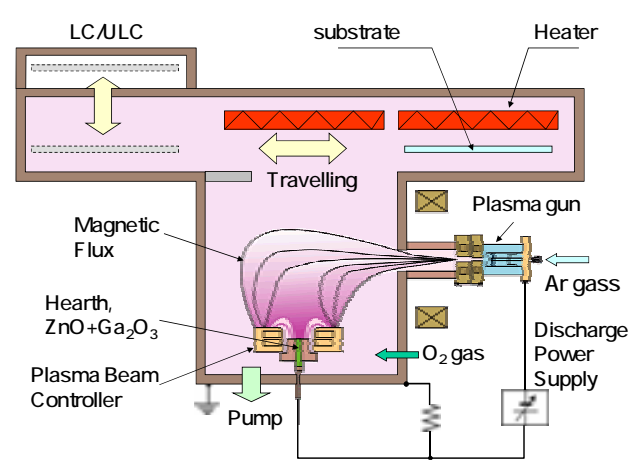

Figure 1. Schematic diagram of ion plating with DC-arc discharge.

The deposition system is composed of a pressure-gradient arc plasma source at the cathode (plasma gun), a plasma beam controller around the anode (hearth) and a substrate holder. The evaporation source is set at the center of the hearth and is thermally evaporated by the DC arc-discharge plasma, which is focused by a magnetic field. The evaporated particles are ionized through the high-density plasma above the hearth before reaching the substrate.

The film thickness $d$ and $T_{s}$ are controlled by changing the traveling speed of the substrate holder. This deposition system does not have a system for cooling the substrate, therefore, $T_{s}$ increases with plasma exposure during the deposition. The evaporation source was sintered ceramic $\mathrm{ZnO}$ (99.99\% pure) containing $4 \mathrm{wt} \%$ $\mathrm{Ga}_{2} \mathrm{O}_{3}(99.9 \%$ pure, HAKUSUI Tech. SKY-Z). The base pressure and deposition pressure were $9.35 \times 10^{-5} \mathrm{~Pa}$ and $0.47 \mathrm{~Pa}$, respectively. During deposition, the flow rates of argon gas (Ar) and oxygen gas $\left(\mathrm{O}_{2}\right)$, which was introduced into the deposition chamber to compensate for the oxygen deficiency, were $240 \mathrm{sccm}$ and $15 \mathrm{sccm}$, respectively.

The discharge current between the plasma gun and the evaporation source set at the center of the hearth was $100 \mathrm{~A}$. Several values of $T_{s}$ less than $100^{\circ} \mathrm{C}$, measured using a thermocouple attached to the surface of the substrate, can be obtained by changing the plasma exposure time $t_{p}$ by controlling the traveling speed of the substrate holder.

Fourteen samples of GZO films with a size of $30 \times 50 \mathrm{~mm}$ were prepared. Details of some samples are summarized in Table 1 . The $d$ was measured using a surface profilometer. The structural analysis was carried out by $\mathrm{X}$-ray diffraction (XRD) measurement $(\mathrm{Cu} K \alpha$ wavelength $\lambda=$ $0.1540562 \mathrm{~nm}$ ) system (Rigaku ATX-G). 
Table 1. Representative sample name, type of substrates, substrate temperature $T_{\mathrm{s}}$, plasma exposure time $t_{\mathrm{p}}$, total number of depositions $N$ and film thickness $d$.

\begin{tabular}{|c|c|c|c|c|c|}
\hline Sample & $\begin{array}{c}\text { type of } \\
\text { substrate }\end{array}$ & $\begin{array}{c}T_{s} \\
{\left[{ }^{\circ} \mathrm{C}\right]}\end{array}$ & $\begin{array}{c}t_{p} \\
{[\mathrm{~s}]}\end{array}$ & $N$ & $\begin{array}{c}d \\
{[\mathrm{~nm}]}\end{array}$ \\
\hline $\mathrm{ZC} 1$ & COP & 50 & 17.5 & 4 & 103 \\
\hline $\mathrm{ZC} 2$ & COP & 60 & 23.3 & 3 & 105 \\
\hline $\mathrm{ZC} 3$ & COP & 70 & 35 & 2 & 104 \\
\hline $\mathrm{ZC} 4$ & COP & 90 & 70 & 1 & 106.5 \\
\hline ZG1 & glass & 50 & 17.5 & 4 & 103 \\
\hline ZG2 & glass & 60 & 23.3 & 3 & 105 \\
\hline ZG3 & glass & 70 & 35 & 2 & 104 \\
\hline ZG4 & glass & 90 & 70 & 1 & 106.5 \\
\hline
\end{tabular}

Electrical resistivity $\rho$, carrier concentration $n$ and Hall mobility $\mu$ of the films on two different substrates were determined by a Hall-effect measurement with the van der Pauw method (Nanometrics, HL5500PC) at room temperature. The optical transmittance of the substrates with the films was measured using a UV/Visible/NIR spectrophotometer (Hitachi, U-4100).

\section{Results and Discussion}

\subsection{Structural Properties}

Figure 2 shows the out-of-plane XRD patterns of ZG1 and ZC1. For all the GZO films, the analysis of data obtained from out-of-plane XRD patterns showed that only one peak at approximately $34.4^{\circ}$ was observed in the $2 \theta$ range from $20^{\circ}$ to $80^{\circ}$. This peak is identified as the (002) diffraction peak from $\mathrm{ZnO}$ with a wurtzite structure. Three diffraction peaks for $\mathrm{ZG} 1$ and $\mathrm{ZC} 1$ were observed, corresponding to the (100), (110) and (200) diffraction peaks of wurtzite $\mathrm{ZnO}$.

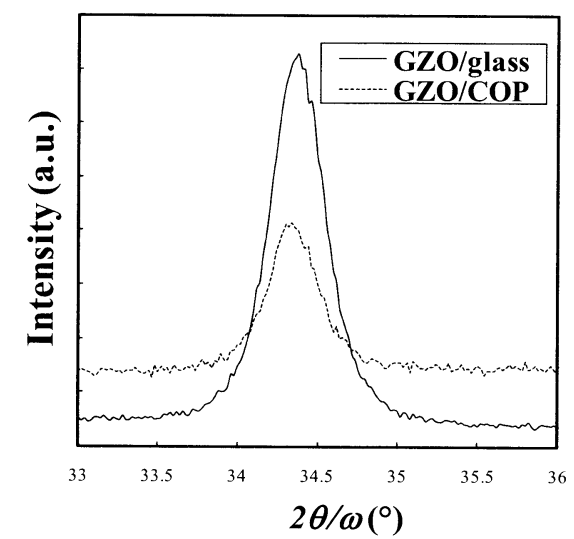

Figure 2. Out-of-plane XRD patterns of ZG1 and ZC1 (see Table1).

The in-plane XRD patterns indicate that the (101) and (201) diffraction peaks of wurtzite $\mathrm{ZnO}$ were observed clearly for the GZO film on the COP substrates (GZO/COP) whereas those peaks were negligible for the GZO films on the glass substrates (GZO/glass). No other phase, such as $\mathrm{Ga}_{2} \mathrm{O}_{3}$, was detected from the XRD analysis. Results similar to those obtained at $T_{s}=50^{\circ} \mathrm{C}$ were obtained at all $T_{s}$.

The above findings show that all the GZO films deposited on the COP or glass substrates at $T_{s}$ below $100^{\circ} \mathrm{C}$ have a strong $c$-axis orientation perpendicular to the substrates. However, for $\mathrm{GZO} / \mathrm{COP}$, the (002) peak intensity was decreased compared with that of GZO /glass at any given $T_{s}$, which indicates that the degree of crystallinity of $\mathrm{GZO} / \mathrm{COP}$ was deteriorated. The high surface roughness of the COP substrate (average surface roughness $R_{a}=1.85 \mathrm{~nm}$ determined by AFM measurements) compared with that of the glass substrate $\left(R_{a}=0.12 \mathrm{~nm}\right)$ may cause the deterioration in the crystallinity of GZO/COP.

\subsection{Electrical Properties}

Figure 3 shows $\rho$ of both GZO/COP and $\mathrm{GZO} /$ glass as a function of $T_{s}$. The $T_{s}$-dependent behaviour of $\rho$ for $\mathrm{GZO} / \mathrm{COP}$ was similar to that of GZO/glass: $\rho$ decreased with $T_{s}$. The values of $\rho$ of GZO /COP were slightly higher than those of $\mathrm{GZO} /$ glass at any given $T_{s}$. In addition, it seems that the difference in $\rho$ between $\mathrm{GZO} / \mathrm{COP}$ and $\mathrm{GZO} / \mathrm{glass}$ increased with $T_{s}$. It is of particular interest to consider the cause of this difference in behavior with $T_{s}$, and the variation in the electrical properties with $T_{s}$ was therefore further investigated in terms of $n$ and $\mu$ from the inversely proportional relationship between $\rho$ and the product of $n$ and $\mu$.

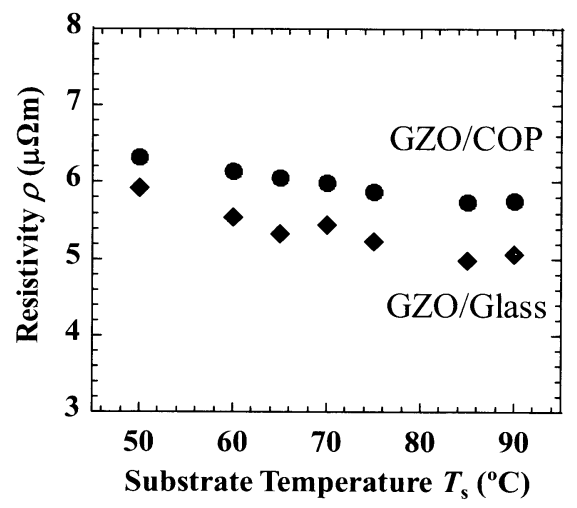

Figure 3. Resistivity $\rho$ of GZO films on COP and glass substrates as a function of substrate temperature $T_{\mathrm{s}}$. 


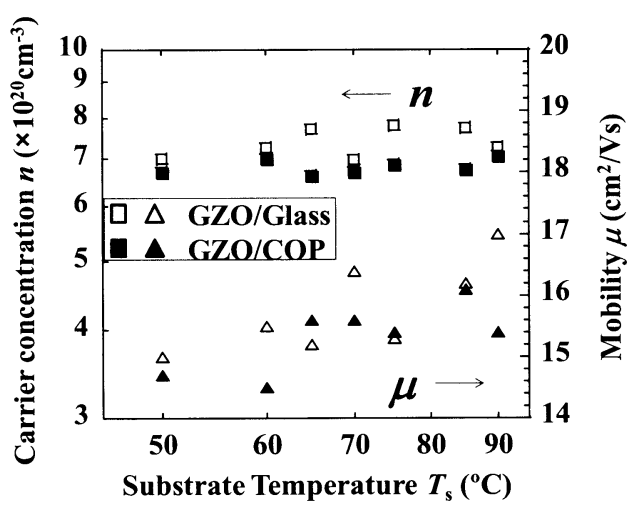

Figure 4. Carrier concentration $n$ and Hall mobility $\mu$ of GZO films on COP and glass substrates as a function of substrate temperature $T_{\mathrm{s}}$.

Figure 4 shows the $T_{s}$ dependences of $n$ and $\mu$ of the GZO films on the two different substrates. For both $\mathrm{GZO} /$ glass and $\mathrm{GZO} / \mathrm{COP}$, with increasing $T_{s}$, $\mu$ increased slowly while $n$ changed negligibly.

The $n$ of $\mathrm{GZO} /$ glass is larger than that of $\mathrm{GZO} / \mathrm{COP}$ at any given $T_{s}$. With increasing $T_{s}$, the $\mu$ of GZO/glass shows a sharp rise compared with that of GZO/COP. As a result, $\rho$ of $\mathrm{GZO} /$ glass decreased rapidly, whereas $\rho$ of $\mathrm{GZO} / \mathrm{COP}$ decreased slightly as $T_{s}$ increased, as shown in Fig. 3.

\subsection{Optical Properties}

Figure 5 shows the representative optical transmittance $(T)$ spectra in the near-ultraviolet (UV) and visible (VIS) regions for the all the samples in Table 1 and the substrates alone. The $T \mathrm{~s}$ of the glass and COP substrates themselves at a wavelength $\lambda$ of $550 \mathrm{~nm}$ were $92 \%$ and $91 \%$, respectively. The two figures show little difference $(0.5-0.7 \%)$ in $T$ among GZO/COP and GZO/glass prepared at different $T_{s}$ at any given $\lambda$.
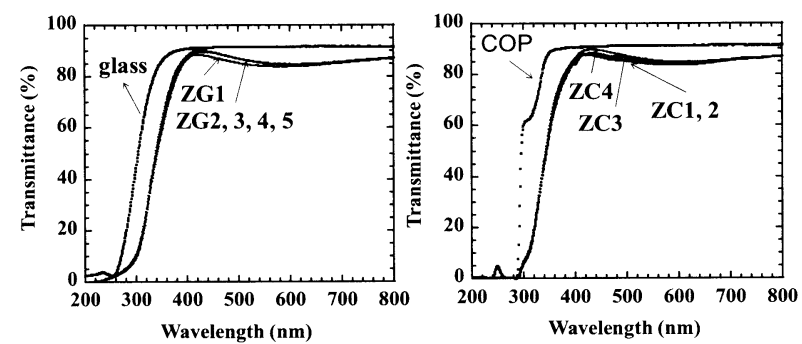

Figure 5. Transmittance spectra in near-ultraviolet and visible regions of the representative samples with GZO films on (a) COP and (b) glass substrates deposited at different substrate temperatures $T_{s}$ (see Table 1).
All the GZO films exhibited high total light transmittance of more than $86 \%$ regardless of $T_{s}$, low absorption in the VIS region, and a sharp fundamental absorption edge in the UV region. In the VIS region, these developed GZO films themselves showed transmission greater than $90 \%$ and a broad dip, which is due to the multiple interference of the light reflected at the surface and at the interface between the GZO thin films with refractive indexes of 1.8-2.0 and the substrates with refractive indexes of approximately 1.51-1.53. The observed interference effect indicates that all the GZO films are reasonably flat.

Next, the optical properties of both $\mathrm{GZO} /$ glass and GZO/COP were investigated in terms of the optical absorption coefficient $\alpha$, using the transmission data in the wavelength range of 380-800nm:

$$
\alpha=\frac{1}{d} \ln \left(\frac{1}{T}\right)
$$

where $d$ is the GZO film thickness and $T$ is the optical transmission, respectively. The results are plotted in Figs. 6(a) and (b). Note that small $\alpha$ values indicate high transmission.

Figures 6(a) and (b) show that GZO films deposited at higher $T_{s}$ had smaller $\alpha$ throughout the observed spectrum. The lowest $\alpha$ values of around $1.2 \times 10^{3} \mathrm{~cm}^{-1}$ were observed for a GZO film deposited at $T_{s}=90^{\circ} \mathrm{C}$ at a wavelength of $423 \mathrm{~nm}$ for $\mathrm{GZO} /$ glass and $427 \mathrm{~nm}$ for $\mathrm{GZO} / \mathrm{COP}$.

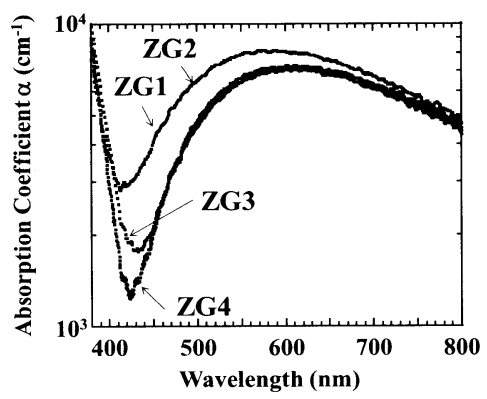

Figure 6(a). Absorption coefficient of GZO/glass (see Table 1) as a function of wavelength.

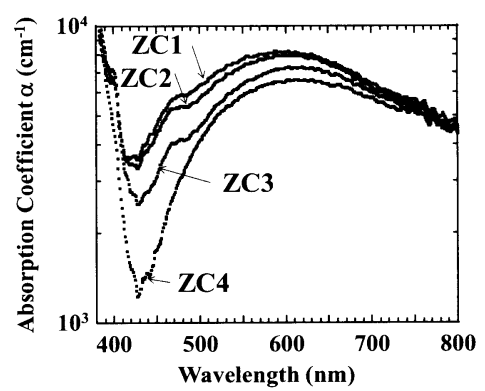

Figure 6(b). Absorption coefficient of GZO/glass (see Table 1) as a function of wavelength 
The $\alpha$ curves exhibit a typical characteristic of TCO, and show the minimum in the VIS region. The reduction of $\alpha$ observed in both $\mathrm{GZO} / \mathrm{glass}$ and $\mathrm{GZO} / \mathrm{COP}$ can be attributed to the increase of $\mu$, as shown in Fig. 4 , because $\alpha$ in the VIS region is inversely proportional to $\mu$, and directly proportional to $n$, which changes little regardless of the type of substrate.

\subsection{Residual Stress}

Figure 7 shows the variation of $c$-axis lattice constants, which was calculated using out-of-plane $\mathrm{XRD}$ data, of $\mathrm{GZO} / \mathrm{COP}$ and $\mathrm{GZO} /$ glass as a function of $T_{s}$. The corresponding values for strain-free GZO powder [11], theoretical calculations using an ab-initio electronic band structure calculation method for GZO [9] and reference $\mathrm{ZnO}$ powder [12] were $5.217 \AA$ (see Fig.

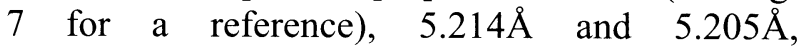
respectively.

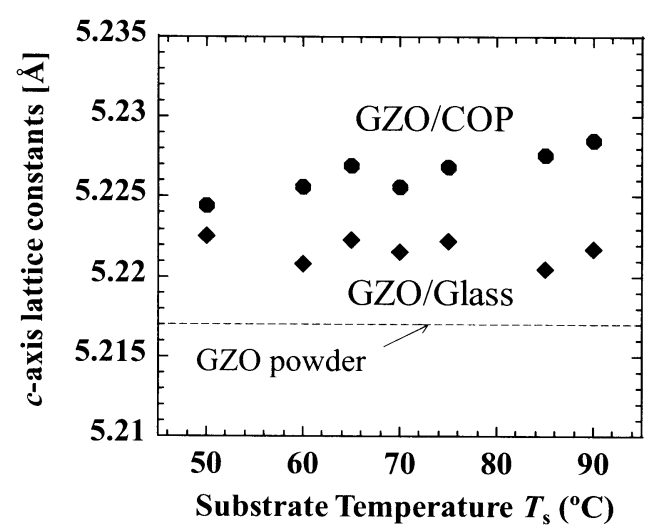

Figure 7. Lattice constant of $c$-axis for GZO/COP and $\mathrm{GZO} /$ glass at various substrate temperatures.

The values of the $c$-axis lattice constants of $\mathrm{GZO} / \mathrm{COP}, c_{\mathrm{GZO} / \mathrm{COP}}$, are larger than thoseof $\mathrm{GZO} /$ glass, $c_{\mathrm{GZO} / \text { glass }}$, at any given $T_{s}$. Figure 7 indicates a distinct deference in $T_{s}$ dependence between $c_{\mathrm{GZO} / \mathrm{COP}}$ and $c_{\mathrm{GZO} / \text { glass }}$ : With $T_{s}, c_{\mathrm{GZO} / \mathrm{COP}}$ increased slowly whereas $c_{\mathrm{GzO} / \text { glass }}$ changed negligibly.

The above findings concerning discrepancy in the lattice constants are due to the variation of residual stress in the films. The stresses in films on a substrate consist basically of two components: the "intrinsic stresses", which depend on a number of factors such as structure and density, and "thermal stresses" originating from a difference in the thermal expansion coefficients (TECs) between the film and the substrate.
The TECs of glass substrates and COP substrates are $3.28 \times 10^{-6}{ }^{\circ} \mathrm{C}^{-1}$ and almost $7 \times 10^{-5}{ }^{\circ} \mathrm{C}^{-1}$, respectively. The TECs, $\alpha_{11}$ and $\alpha_{33}$, of hexagonal $\mathrm{ZnO}$ are 6.05 and $3.53 \times 10^{-6}{ }^{\circ} \mathrm{C}^{-1}$, respectively [13], $\alpha_{i j}$ is the tensor element. Thus, the residual stress in $\mathrm{GZO} /$ glass is the intrinsic stress, whereas the thermal stresses arising during the variation of temperature owing to the difference in the thermal expansion coefficients between the GZO film and the substrate are significant in GZO/COP.

The residual stress was evaluated from the XRD spectrum. The calculation of the wurtzite GZO film stress is based on the biaxial strain model $[14,15]$. The residual strain normal to the substrate is given by $\varepsilon=\left(c_{\text {film }}-c_{\text {bulk }}\right) / c_{\text {bulk }}$ along the $c$-axis (where $c_{\text {film }}$ and $c_{\text {bulk }}$ are the $c$-axis lattice constant of the film and the strain-free lattice $c$-axis constant of GZO powder, respectively). $c_{\text {film }}$ was calculated using out-of-plane XRD data.

To evaluate the residual stress $\sigma_{G Z O . f}^{X R D}$ in GZO films, derived from XRD, parallel to the film surface, the following formula which is valid for a hexagonal lattice $[14,15]$ is used,

$$
\sigma_{G Z O . f}^{X R D}=\frac{2 c_{13}^{2}-c_{33}\left(c_{11}+c_{12}\right)}{2 c_{13}} \frac{c_{G Z O . f}-c_{G Z O . p o w}}{c_{G Z O . p o w}}
$$

For the elastic constants $c_{i j}$ and $c_{G Z o . p o w}$, the following values for single-crystal $\mathrm{ZnO}$ and strain-free GZO powder described above was used: $c_{11}=208.8, c_{33}=213.8, c_{12}=119.7, c_{13}=104.2$ $\mathrm{GPa}[16]$.

Residual stress calculated from eq. (2) using $\mathrm{XRD}$ data, in both GZO/COP and GZO/glass as a function of $T_{s}$ are shown in Fig. 8.

All the GZO films prepared by ion-plating deposition show evidence of a compressive stress as do sputtered $[15,17,18]$.

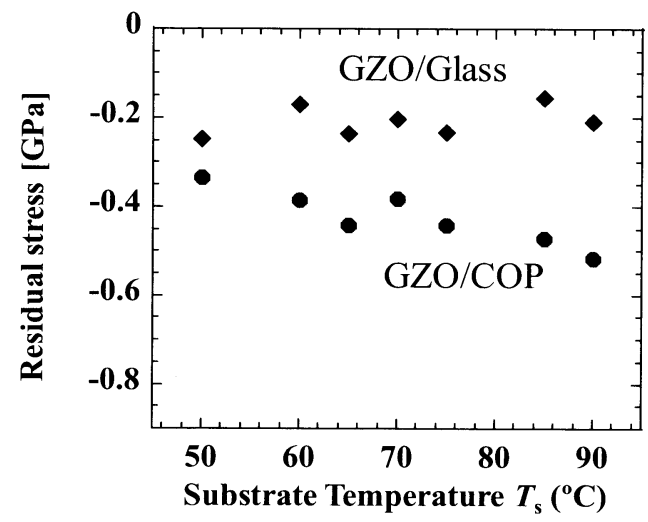

Figure 8. Lattice constant of $c$-axis for GZO/COP and $\mathrm{GZO} /$ glass at various substrate temperatures. 
The residual stresses in GZO/glass are very low and their $T_{s}$-dependent curves exhibit a typical characteristic of intrinsic stress, that is, a decreasing function of $T_{s}$. This $T_{s}$ dependence of the residual stress is similar to that of GZO film conductivity, as shown in Fig. 3.

On the other hand, for $\mathrm{GZO} / \mathrm{COP}$, Fig. 8 indicates that the residual stress is an increasing function of $T_{s}$ and reached almost $0.5 \mathrm{GPa}$ at $T_{s}$ $=90^{\circ} \mathrm{C}$. Considering the behavior of the $c$-axis lattice constants of GZO films on the two different substrates against $T_{s}$ described above, as shown in Fig. 7, when the rise of $T_{s}$ during deposition is less than $60^{\circ} \mathrm{C}$, the main residual stresses are probably intrinsic stress arising from the film density and structure. At higher temperatures, thermal stress is dominant.

\section{Summary}

Highly transparent conductive crack-free GZO films with a thickness of almost $100 \mathrm{~nm}$ on COP and alkali-free glass substrates have been obtained at $T_{s}$ of less than $100{ }^{\circ} \mathrm{C}$. GZO films were prepared by ion-plating deposition with $\mathrm{DC}$ arc discharge. Various values of $T_{s}$ of less than $100^{\circ} \mathrm{C}$ were obtained by controlling the plasma exposure time.

The analysis of the XRD patterns of the films showed that all polycrystalline GZO films on the two different substrates have a preferred orientation along the $c$-axis regardless of $T_{s}$. The crystallity of GZO/COP is lower than that of GZO /glass at any given $T_{s}$.

It was found that the resistivity of GZO films on the two different substrates decreases with $T_{s}$ and that the resistivity of $\mathrm{GZO} / \mathrm{COP}$ is greater than that of GZO/glass at any given $T_{s}$.

The total light transmittance of all the GZO films on the two different substrates was more than $86 \%$ regardless of $T_{s}$. A small difference in the transmittance between $\mathrm{GZO} / \mathrm{COP}$ and $\mathrm{GZO} /$ glass is observed at any given $\lambda$ in the VIS region.

All the GZO films have compressive stress. When the deposition is performed at a low temperature of less than $60^{\circ} \mathrm{C}$, intrinsic stress contributes to the residual stress regardless of the type of substrate. For GZO/COP deposited at $T_{s}$ above $60^{\circ} \mathrm{C}$, thermal stress contributes mainly to the residual stress.

\section{Acknowledgement}

This work is supported by the Ministry of Economy, Trade and Industry (2007) and New Energy and Industrial Technology Development Organization (NEDO) (2008 ) under the National
Project of Rare Metal Indium (In) Substitute Materials Development.

\section{References}

1. B.-S. Chiou and S.-T. Hsieh, Thin Solid Films, 229 (1993), 146.

2. X. Hao, J. Maa, D. Zhanga, T. Yang, H. Maa, Y. Yanga, C. Chengc and J. Huang, Appl. Surf. Sci., 183 (2001), 137.

3. T. Yamamoto, T. Yamada, A. Miyake, H. Makino and N. Yamamoto, IEICE Trans., E91-C (2008), 1547.

4. T. Yamamoto, T. Yamada, A. Miyake, H. Makino and N. Yamamoto, J. Soc, Info. Display., 16/7 (2008), 713

5. J. N. Bardsley, ed., M. Robert Pinnel, USDC Flexible Display Report, U.S. Display Consortium, 2004.

6. T. Kuno, T. Kawamura and K. Nitta, Polym. Bull., 59 (2008), 847.

7. M. Brett, R. McMahon, J. Affinito and S. Parson, J. Vac. Sci. Technol., A1 (1984), 162.

8. H. Hirasawa, M. Yoshida, S. Nakamura, Y. Suzuki, S. Okada and K. Kondo, Solar Energy Materials and Solar Cells, 67 (2001), 231.

9. T. Yamamoto, T. Sakemi, K. Awai and S. Shirakata, Thin Solid Films, 451-452 (2004), 439.

10. T. David, S. Goldsmith and R L Boxman, J. Phys. D: Appl. Phys., 38 (2005), 2407.

11. R. Wang, A. W. Sleight and D. Cleary, Chem. Mater., 8 (1996), 433.

12. W. T. Lim and C. H. Lee, Thin Solid Films, 353 (1999), 12.

13. J. H. Joe, M. Y. Han and D. J. Cheng, J. Appl. Phys., 71 (1992), 4333.

14. R. W. Hoffman, "Physics of Nonmetallic Thin Films", Eds. C. H. S. Dupy and A. Cachard, New York, 1976, p. 273.

15. S. Maniv, W. D. Westwood and E. Colombini, J. Vac. Sci. Technol., 20(2) (1982), 162.

16. R. Cebulla, R. Wendt and K. Ellmer, $J$. Appl. Phys., 83 (1998), 1087.

17. R. Hong, J. Shao, H. He and Z. Fan, J. Crystal Growth, 284 (2005), 347.

18. Z. B. Fang, Z. J. Yan, Y. S. Tan, X. Q. Liu and Y. Y. Wang, Appl. Surf. Sci., 241 (2005) 303. 